\title{
PHD Finger Protein 19 Promotes Cardiac Hypertrophy via Epigenetically Regulating SIRT2
}

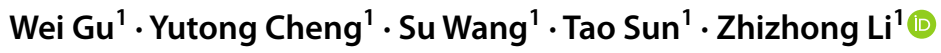

Received: 5 November 2020 / Accepted: 27 January 2021 / Published online: 21 February 2021

(c) The Author(s) 2021

\begin{abstract}
Epigenetic regulations essentially participate in the development of cardiomyocyte hypertrophy. PHD finger protein 19 (PHF19) is a polycomb protein that controls H3K36me3 and H3K27me3. However, the roles of PHF19 in cardiac hypertrophy remain unknown. Here in this work, we observed that PHF19 promoted cardiac hypertrophy via epigenetically targeting SIRT2. In angiotensin II (Ang II)-induced cardiomyocyte hypertrophy, adenovirus-mediated knockdown of Phf19 reduced the increase in cardiomyocyte size, repressed the expression of hypertrophic marker genes Anp and Bnp, as well as inhibited protein synthesis. By contrast, Phf19 overexpression promoted Ang II-induced cardiomyocyte hypertrophy in vitro. We also knocked down Phf19 expression in mouse hearts in vivo. The results demonstrated that Phf19 knockdown reduced Ang II-induced decline in cardiac fraction shortening and ejection fraction. Phf19 knockdown also inhibited Ang II-mediated increase in heart weight, reduced cardiomyocyte size, and repressed the expression of hypertrophic marker genes in mouse hearts. Further mechanism studies showed that PHF19 suppressed the expression of SIRT2, which contributed to the function of PHF19 during cardiomyocyte hypertrophy. PHF19 bound the promoter of SIRT2 and regulated the balance between $\mathrm{H} 3 \mathrm{~K} 27 \mathrm{me} 3$ and H3K36me3 to repress the expression of SIRT2 in vitro and in vivo. In human hypertrophic hearts, the overexpression of PHF19 and downregulation of SIRT2 were observed. Of importance, PHF19 expression was positively correlated with hypertrophic marker genes ANP and BNP but negatively correlated with SIRT2 in human hypertrophic hearts. Therefore, our findings demonstrated that PHF19 promoted the development of cardiac hypertrophy via epigenetically regulating SIRT2.
\end{abstract}

Keywords Cardiac hypertrophy $\cdot$ Epigenetics $\cdot$ PHF19 $\cdot$ Histone methylation $\cdot$ SIRT2

\section{Introduction}

Cardiovascular diseases have become the first leading cause of death worldwide [1-4]. Various cardiovascular risk factors can lead to cardiac hypertrophy, one of the fundamental mechanisms underlying multiple heart diseases. In general, hypertension, myocardial infarction, and neuroendocrine factors can lead to cardiac hypertrophy. Cardiac hypertrophy is classified as physiological and pathological hypertrophy. Physiological hypertrophy of the hearts occurs during

Handling Editor: Y. James Kang.

Zhizhong Li

lzzeagle@sina.com

1 Department of Cardiology, Beijing Anzhen Hospital, Capital Medical University and Beijing Institute of Heart Lung and Blood Vessel Diseases, 2 Anzhen Road, Beijing, China development or pregnancy, and this type of hypertrophy is revisable [5]. Under pathological conditions, the cardiomyocytes cannot proliferate to support the decreased cardiomyocyte numbers or increased demand for overload. Instead, the cardiomyocytes undergo pathologically hypertrophic growth. However, this pathological hypertrophy is irreversible and can finally result in heart failure and arrhythmia [6-8]. The understanding of the mechanisms underlying pathological hypertrophy is critical for the treatment of cardiac diseases.

During the past decade, accumulating studies have demonstrated the critical roles of epigenetic regulators in cardiac hypertrophy. Histone acetylation and methylation are critically involved in the development of cardiac hypertrophy [5]. For instance, Jumonji domain-containing 2A (JMJD2A) promoted the development of pathological cardiac hypertrophy via regulating $\mathrm{H} 3 \mathrm{~K} 9 \mathrm{me} 3$ and expression of four-and-ahalf LIM domains 1 [9]. The histone demethylase JMJD1C 
was also reported to modulate CAMKK2-AMPK signaling to contribute to cardiac hypertrophy [10]. H3K9me2-specific demethylase KDM3A promoted cardiac hypertrophy and fibrosis in response to pressure-overload. Cardiomyocyte KDM3A activated Timp1 transcription to promote cardiac remodeling [11]. Histone acetylation was also reported to participate in cardiac hypertrophy. Class I histone deacetylase (HDAC) was demonstrated to promote cardiac hypertrophy, and inhibitors of class I HDACs are potential drugs for the treatment of cardiac hypertrophy [6, 12].

PHD finger protein 19 (PHF19) is a polycomb protein that controls H3K36me3 and H3K27me3. For instance, PHF1 9 bound $\mathrm{H} 3 \mathrm{~K} 36 \mathrm{me} 3$ to recruit PRC2 and demethylase NO66 to embryonic stem cell genes during differentiation [13]. PHF19 also controlled hematopoietic stem cell state and differentiation [14]. In multiple myeloma, PHF19 promoted tumorigenicity through PRC2 activation and broad H3K27me3 domain formation [15, 16]. Besides, PHF19 also regulated the proliferation and invasiveness of prostate cancer cells via H3K27me3 deposition [17]. However, the roles of PHF19 in the cardiovascular system remain unknown.

SIRT2 belongs to the class III histone deacetylase Sirtuin family. SIRT2 can locate in the cytoplasm, nucleus, and mitochondria and controls the deacetylation of histones, cytoplasmic, and mitochondrial proteins [18]. Several studies have reported the critical roles of SIRT2 in pathological hypertrophy [19-21]. For instance, Tang et al. reported that SIRT2 repressed the development of aging-related cardiac hypertrophy via deacetylating LKB1 and activating the LKB1-AMPK signaling pathway [19]. However, the upstream regulators of SIRT2 expression during cardiac hypertrophy remain unknown.

In the present study, we identified that PHF19 promoted cardiac hypertrophy via downregulating SIRT2. The expression of PHF19 was upregulated in hypertrophic hearts. Overexpression of PHF19 promoted cardiomyocyte hypertrophy in vitro and in vivo. Mechanism study revealed that PHF19 epigenetically repressed the expression of SIRT2 via controlling H3K36me 3 and H3K27me3 switch. Finally, we observed that SIRT2 was critically involved in PHF19 function in cardiomyocyte hypertrophy.

\section{Materials and Methods}

\section{Human Heart Samples}

All human heart samples were collected at Beijing Anzhen Hospital, Capital Medical University, from 2016 to 2020. Before use, the samples were collected and stored at $-80^{\circ} \mathrm{C}$. The study was approved by the Ethics Committee of Beijing Anzhen Hospital, and every patient has signed the informed consent.

\section{Animal Models}

Cardiac hypertrophy in 8-12-week-old male C57BL/6 mice were induced by chronic infusion of angiotensin II (Sigma, A9525, $1.5 \mathrm{mg} / \mathrm{kg} /$ day) with Alzet $2004 \mathrm{~min}$ ipump for 4 weeks. The ejection fraction and fraction shortening were monitored by echocardiography. Heart rate and blood pressure were also measured at the end of the experiment. Heart weight, body weight, and tibia length were also analyzed. The animal study was approved by the Animal Study Ethics Committee of Beijing Anzhen Hospital.

\section{Isolation of Neonatal Rat Cardiomyocytes}

Primary cardiomyocytes were isolated from neonatal (1-3 day) Sprague Dawley rat left ventricles, as described previously [22]. The neonatal rat cardiomyocytes were cultured in DMEM (Gibco) supplemented with 10\% FBS (Gibco), in the presence of BrdU (Sigma), at the condition of $37{ }^{\circ} \mathrm{C}$ and $5 \% \mathrm{CO}_{2}$.

\section{Adenovirus and Adeno-Associated Virus}

The adenovirus system was used for overexpression or knockdown of Phf19 in rat cardiomyocytes. In brief, shPhf19 (5'-GGAAAGAGCAAGCCTGGTTTG-3'), Phf19-overexpressing, and respective control constructs were cloned into adenovirus-packaging plasmids, and the adenovirus was packaged in HEK293A cells, as described previously [23]. For the knockdown of Phfl9 in mouse hearts, an adenoassociated virus 9 (AAV9) system was applied, as described previously [24]. AAV9 expressing shPhf19 (5'-GCTTGC ACACAGTGCCTTAGT-3') or control shRNA was obtained and injected into neonatal mice. The neonatal mice received different AAV9 $\left(3 \times 10^{12}\right.$ vg per mouse $)$ via systemic administration into the temporal vein using a $30-G$ needle. Each mouse received a single dose of AAV9-shRNA. Eight weeks later, the mice were subjected to cardiac hypertrophy induction with Ang II.

\section{In Vitro Model of Cardiomyocyte Hypertrophy}

For cardiomyocyte hypertrophy in vitro, cardiomyocytes were pretreated with $1 \%$ FBS overnight and then treated with Ang II $(1 \mu \mathrm{M})$ for $48 \mathrm{~h}$. The cardiomyocytes were stained with a-actinin antibody (Sigma), and cardiomyocyte size of 50 cells per group was analyzed with Image $\mathbf{J}$ via a blinded fashion. The average cardiomyocyte sizes of three independent experiments were used for analysis. For inhibition of 
SIRT2, the cells were treated with SIRT2 inhibitor AGK2 $(10 \mu \mathrm{M})$ or DMSO.

\section{Quantitative Real-Time PCR}

For quantitative real-time PCR, the SYBR Green master mix kit (ThermoFisher) was applied. Briefly, total RNA was obtained from cells or tissues with TRIzol reagent (Invitrogen), and the first-strand cDNA was generated with the cDNA Synthesis Kit (Sigma), followed by qRT-PCR. The primers used in this study are listed in Table 1.

\section{Western Blot}

Heart tissues and cardiomyocytes were subjected to protein isolation with RIPA reagent. The protein samples were treated with protease inhibitors to reduce degeneration. 40 ug of total protein was subjected to western blot with the protocol described previously [25]. The following antibodies were used: anti-PHF19 antibody (Cell Signaling Technology), anti-SIRT2 antibody (Proteintech), anti-GAPDH (Protein tech).

\section{Chromatin Immunoprecipitation}

To analyze the enrichment of proteins at SIRT2 promoter, chromatin immunoprecipitation (ChIP) was performed with IgG, PHF19, H3K36me3, H3K27me3 antibodies using the Pierce $^{\mathrm{TM}}$ Agarose ChIP Kit (ThermoFisher). The antibodies for ChIP assay were purchased from Abcam. The relative enrichment of each protein was analyzed by qRT-PCR and normalized by Input.

\section{Protein Synthesis Assay}

To analyze protein synthesis, the incorporation of $\left[{ }^{3} \mathrm{H}\right]$-leucine was monitored as described previously [21]. The cells were treated as described in figure legends, and $\left[{ }^{3} \mathrm{H}\right]$-leucine was added two hours before testing.

\section{Histochemical Analysis}

For histochemical analysis, the H\&E staining was performed with the kit. Briefly, heart tissues were fixed in $4 \%$ paraformaldehyde overnight and then embedded in paraffin. The tissues were cut into 5 um sections. The sections were applied to H\&E staining with the H\&E Staining Kit (Abcam) according to the manual. Cardiomyocyte size was quantified with Image $\mathrm{J}$.

\section{Statistical Analysis}

All experiments were repeated at least three times, and the values are shown as mean $\pm S D$. For the analysis of two group data, Student's $t$ test was applied. For analysis of more than two groups, one-way ANOVA followed by Tukey post hoc test was applied. All statistical analysis was performed with GraphPad Prism 7.

\section{Results}

\section{PHF19 Promotes Cardiomyocyte Hypertrophy In Vitro}

Firstly, we generated adenovirus to knock down Phf19 in neonatal rat cardiomyocytes to investigate the roles of
Table 1 Primers used in this study

\begin{tabular}{lll}
\hline Gene symbol & Forward primer $\left(5^{\prime}\right.$-3') & Reverse primer $\left(5^{\prime}\right.$ '-3') \\
\hline Human ANP & AGCGGACTGGGCTGTAACAG & ACGACGCCAGCATGAGCTCCTTC \\
Human BNP & TGGAAACGTCCGGGTTACAG & CTGATCCGGTCCATCTTCCT \\
Human PHF19 & ACTCGGGACTCCTATGGTGC & CCTCCGTCAGTTTGGACATCA \\
Human SIRT2 & TGCGGAACTTATTCTCCCAGA & GAGAGCGAAAGTCGGGGAT \\
Human GAPDH & GGAGCGAGATCCCTCCAAAAT & GGCTGTTGTCATACTTCTCATGG \\
Mouse Anp & GTGCGGTGTCCAACACAGAT & TCCAATCCTGTCAATCCTAC \\
Mouse Bnp & GAGGTCACTCCTATCCTCTGG & GCCATTTCCTCCGACTTTTCT \\
Mouse Phf19 & GGCCTGGGTTACCACCAAC & CGTCGGCAGAACCAATGAG \\
Mouse Sirt2 & GCGGGTATCCCTGACTTCC & CGTGTCTATGTTCTGCGTGTAG \\
Mouse Gapdh & TGGATTTGGACGCATTGGTC & TTTGCACTGGTACGTGTTGAT \\
Rat Anp & GCTTCCAGGCCATATTGGAG & GCTTCCAGGCCATATTGGAG \\
Rat Bnp & GAGGTCACTCCTATCCTCTGG & GCCATTTCCTCCGACTTTTCT \\
Rat Phf 19 & TCTGCCGACGCTGCATTTT & CAGCACCATTTTCACTGCCTG \\
Rat Sirt 2 & CCACGGCACCTTCTACACATC & CACCTGGGAGTTGCTTCTGAG \\
Rat Gapdh & AGGTCGGTGTGAACGGATTTG & TGTAGACCATGTAGTTGAGGT \\
\hline
\end{tabular}


A

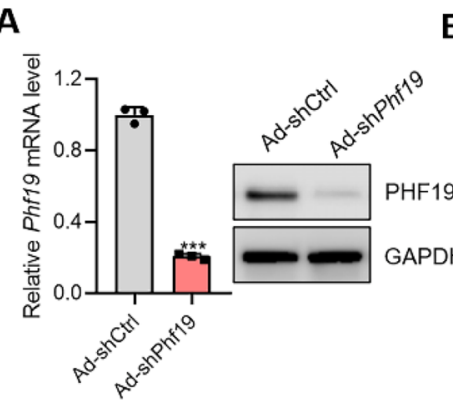

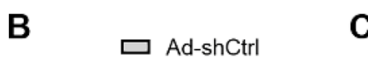

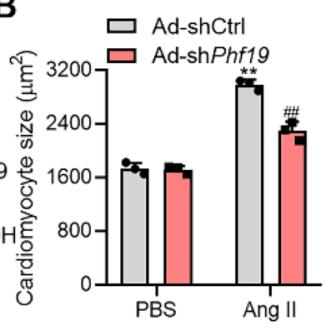

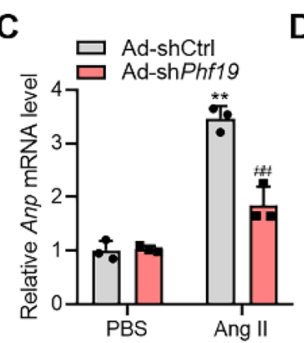
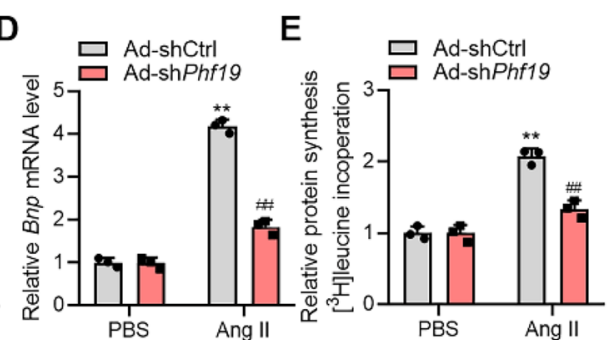

$\mathbf{F}$

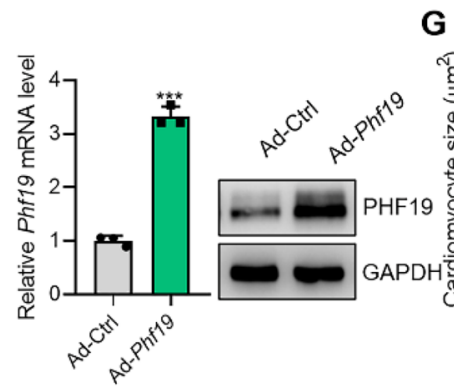

G 口 Ad-Ctrl
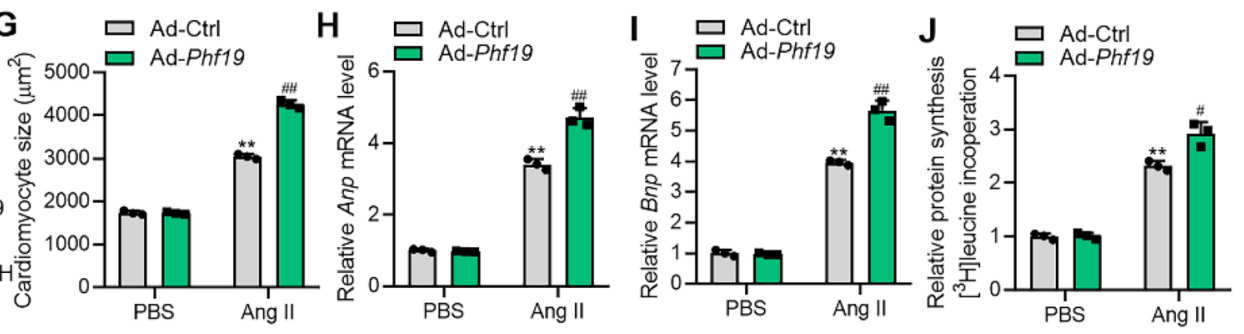

Fig. 1 PHF19 promotes cardiomyocytes hypertrophy in vitro. a Knockdown of Phf19 expression in cardiomyocytes. Neonatal rat cardiomyocytes were infected with adenovirus carrying shRNA targeting Phf19 (Ad-shPhf19) or control shRNA (Ad-shCtrl) $(n=3)$. Data are shown as mean \pm SD. $* * * P<0.001$ versus Ad-shCtrl. b-d Knockdown of Phf19 represses cardiomyocyte hypertrophy $(n=3)$. Neonatal rat cardiomyocytes were infected with Ad-shPhf19 or Ad-shCtrl for $24 \mathrm{~h}$, and then treated with angiotensin II (Ang II, $1 \mu \mathrm{M})$ for $48 \mathrm{~h}$ to induce hypertrophy. b Quantification of cardiomyocyte size. c mRNA level of atrial natriuretic peptide (Anp). d mRNA level of brain natriuretic peptide (Bnp). Data are shown as mean \pm SD. $* * P<0.01$ versus Ad-shCtrl + PBS, \#\#P<0.01 versus Ad-shCtrl + Ang II. d Knockdown of Phf19 represses protein synthesis in cardiomyocytes $(n=3)$. Protein synthesis was monitored with the incorporation of $\left[{ }^{3} \mathrm{H}\right]$-leucine. Data are shown as mean $\pm \mathrm{SD}$.
** $P<0.01$ versus shCtrl +PBS, \#\#P<0.01 versus Ad-shCtrl+Ang II. e Overexpression of Phf19 expression in cardiomyocytes $(n=3)$. Neonatal rat cardiomyocytes were infected with adenovirus carrying rat $P h f 19$ (Ad-Phf19) or control adenovirus (Ad-Ctrl). Data are shown as mean $\pm \mathrm{SD}$. $* * * P<0.001$ versus Ad-Ctrl. f-h Overexpression of Phf19 promotes cardiomyocyte hypertrophy $(n=3)$. Neonatal rat cardiomyocytes were infected with Ad-Phf19 or Ad-Ctrl for $24 \mathrm{~h}$, and then treated with Ang II $(1 \mu \mathrm{M})$ for $48 \mathrm{~h}$ to induce hypertrophy. f Overexpression of Phfl9 expression in cardiomyocytes. g Quantification of cardiomyocyte size. $\mathbf{h}$ mRNA level of Anp. i mRNA level of Bnp. Data are shown as mean \pm SD. $* * P<0.01$ versus Ad-Ctrl + PBS, $\# \# P<0.01$ vs. Ad-Ctrl + Ang II. j Overexpression of Phf19 promotes protein synthesis in cardiomyocytes. Protein synthesis was monitored with the incorporation of $\left[{ }^{3} \mathrm{H}\right]$-leucine. Data are shown as mean $\pm \mathrm{SD}$. $* * P<0.01$ versus Ad-Ctrl + PBS, \#\#P $<0.01$ versus Ad-Ctrl + Ang II

protein synthesis (Fig. 1f-h, Supplementary Figure 1). Therefore, these findings demonstrated that PFH19 enhanced the hypertrophic response to stress stimuli (AngII) in vitro.

\section{PHF19 Promotes Cardiac Hypertrophy In Vivo}

Next, we tested the functional importance of PHF19 in cardiac hypertrophy in vivo. To this end, we silenced the expression of Phf19 in mouse hearts with adeno-associated virus 9-mediated shRNA (AAV9-shPhf19). Neonatal mice were injected with a single dose of AAV9-shCtrl or AAV9-shPhf19, and the knockdown efficiency was tested eight weeks later. Compared with the control AAV9-shCtrl, AAV9-shPhf19 reduced the expression level of Phf19 in mouse hearts significantly (Fig. 2a). Mice received AAV9shCtrl or AAV9-shPhf19 were chronically infused with Ang II for four weeks to induce pathological cardiac hypertrophy. Ang II treatment caused declines in cardiac function parameters, fraction shortening, and ejection fraction. Besides, 

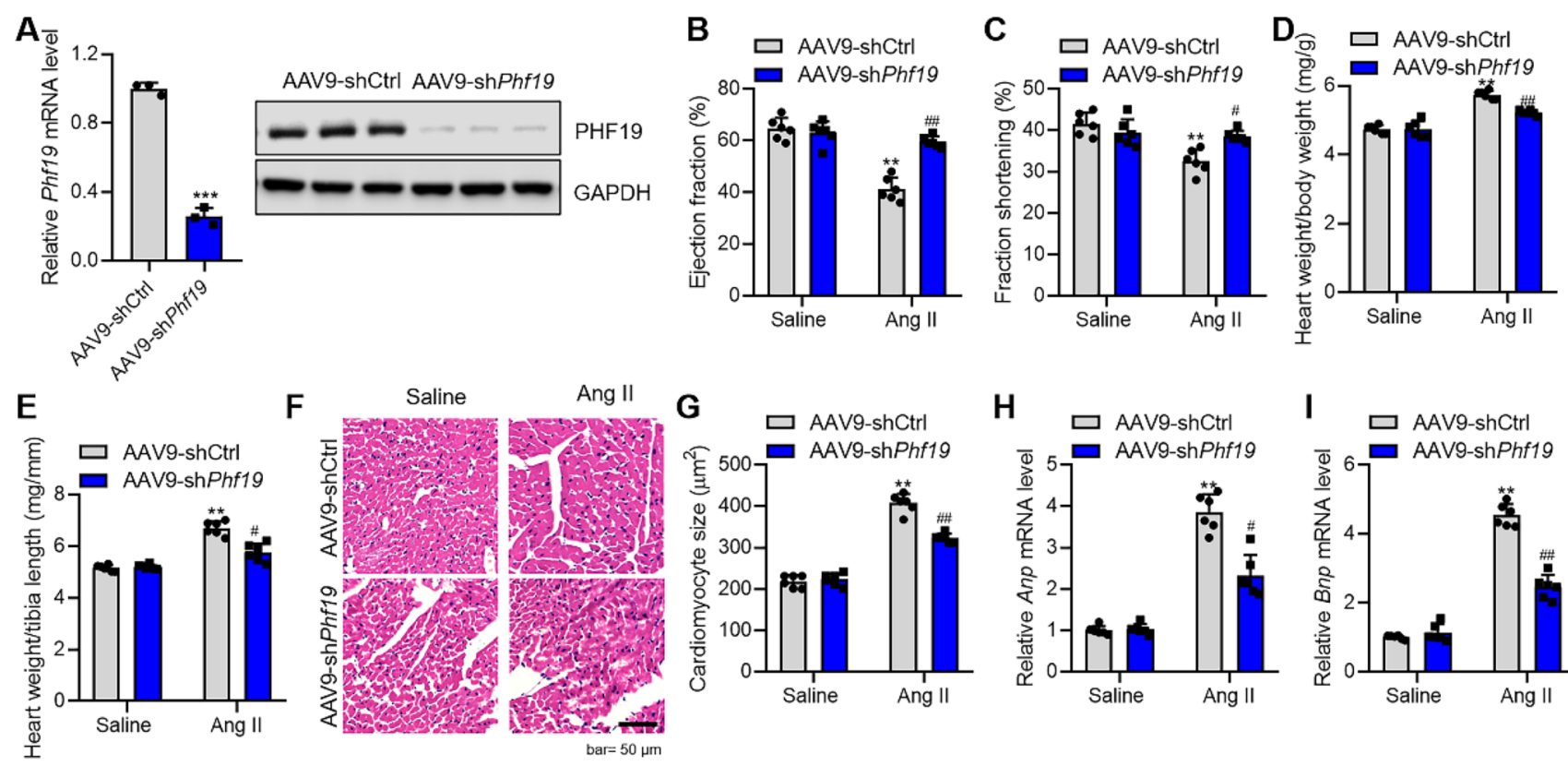

Fig. 2 PHF19 promotes cardiac hypertrophy in vivo. a Phf19 knockdown in cardiac tissues in vivo. Neonatal mice were treated with a single dose of adeno-associated virus 9 (AAV9) carrying shCtrl (AAV9-shCtrl) or shPhf19 (AAV9-shPhf19). Data are shown as mean \pm SD. $n=5$ in each group. ${ }^{* * *} P<0.001$ versus AAV9-shCtrl. b-c Phf19 knockdown represses Ang II-induced decline in cardiac function. Mice with/without Phf19 knockdown were subjected to Ang II treatment for four weeks, and then fraction shortening and ejection fraction were analyzed. Data are shown as mean \pm SD. $n=6$ in each group. ${ }^{* *} P<0.01$ versus AAV9-shCtrl+Saline, \#\#P<0.01 versus AAV9-shCtrl+Ang II. d-e Phf19 knockdown represses Ang II-induced increase in heart weight. Heart weight-to-body weight

Ang II treatment increased heart weight, cardiomyocyte size, and the expression of hypertrophic fetal genes Anp and Bnp. Interestingly, we observed that Phf19 knockdown repressed the decline in cardiac function in mice with cardiac hypertrophy (Fig. 2b and c). In addition, Phfl 19 knockdown repressed Ang II-induced increase in heart weight, cardiomyocyte size, and hypertrophic fetal genes (Fig. 2d-i). However, we did not observe the effects of PHF19 on heart rate and blood pressure (Supplementary Figure 2). Therefore, these findings demonstrated that $P h f 19$ knockdown inhibited cardiac hypertrophy in vivo.

\section{SIRT2 is Involved in the Function of PHF19}

Our findings demonstrated that PHF19 promoted cardiomyocyte hypertrophy in vitro and cardiac hypertrophy in vivo. Then, we explored the potential mechanism underlying PHF19 function during cardiomyocyte hypertrophy. Sirtuins are class III histone deacetylases, which play significant roles in cardiac hypertrophy [26]. We first tested the effects of Phfl9 on the expression of the members of ratio (d) and heart weight to tibia length (e) are shown. Data are shown as mean \pm SD. $n=6$ in each group. $* * P<0.01$ versus AAV9shCtrl+Saline, \#\#P<0.01 versus AAV9-shCtrl+Ang II. f-g Phf19 knockdown represses Ang II-induced increase in cardiomyocyte size. Representative images (f) and quantitative results (g) are shown. Data are shown as mean \pm SD. $n=6$ in each group. $* * P<0.01$ versus AAV9-shCtrl + Saline, \#\#P<0.01 versus AAV9-shCtrl+ Ang II. h-i Phf19 knockdown represses Ang II-induced increase in expression of hypertrophic fetal genes. The expression of Anp (h) and Bnp (i) were analyzed by qRT-PCR. $n=6$ in each group. Data are shown as mean \pm SD. ${ }^{* *} P<0.01$ versus AAV9-shCtrl + Saline, $\# \# P<0.01$ versus AAV9-shCtrl+ Ang II

Sirtuins. We observed that Phf19 knockdown significantly upregulated the expression of Sirt2 but not the other members of the Sirtuins, in rat cardiomyocytes (Fig. 3a). By contrast, Phf19 overexpression reduced the expression of Sirt2 in cardiomyocytes (Fig. 3b). During cardiac hypertrophy, the level of Sirt2 was decreased in the heart tissues. Interestingly, Phf19 knockdown increased the expression of Sirt2 in heart tissues (Fig. 3c and d).

Next, we tested whether the upregulation of Sirt 2 contributed to the anti-hypertrophic roles of Phfl9 shRNA. To this end, SIRT2 was inhibited with a selective inhibitor AGK2 in cardiomyocytes. In Ang II-induced hypertrophic cardiomyocytes, AGK2 treatment significantly promoted hypertrophic growth of cardiomyocytes and repressed the expression of hypertrophic fetal genes and protein synthesis. Significantly, AGK2 treatment blocked the effects of Phf19 knockdown on cardiomyocyte hypertrophy and protein synthesis (Fig. 3e-g, Supplementary Figure 3). Taken together, Phf19 repressed Sirt 2 expression, which partially contributed to the function of Phf19 during cardiomyocyte hypertrophy. 
A

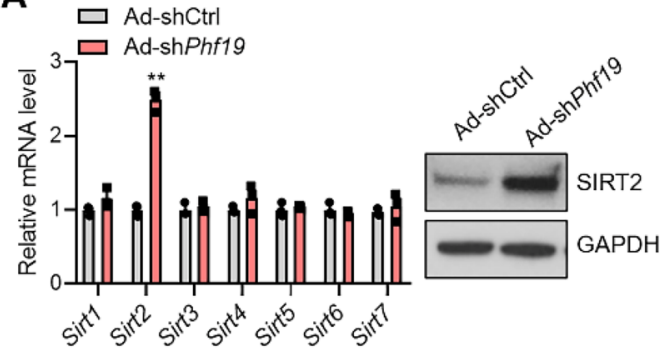

D

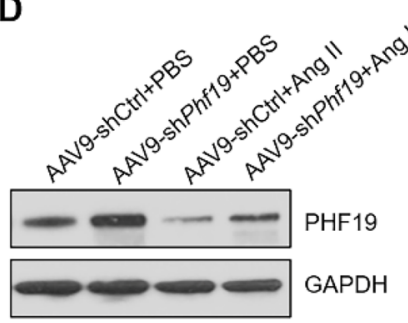

B

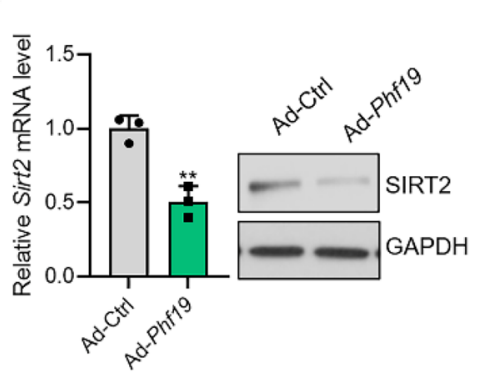

C

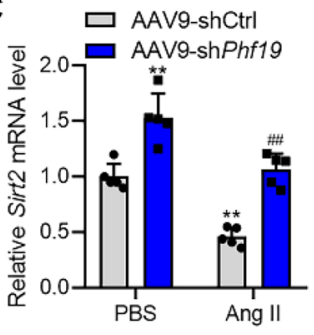

E

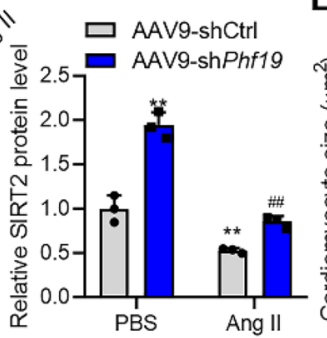

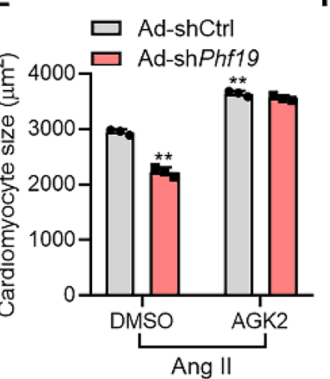

$\mathbf{F}$

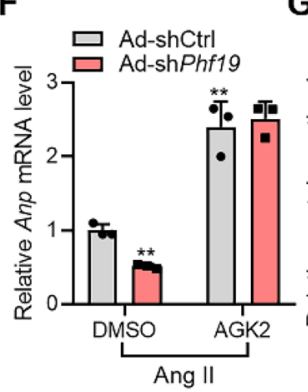

G

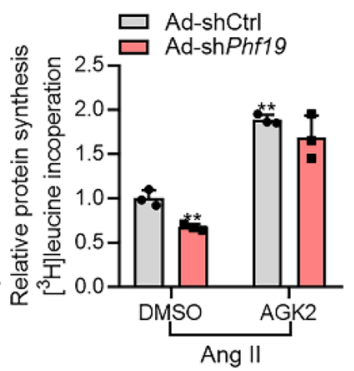

Fig. 3 PHF19 regulates the expression of SIRT2 in cardiomyocytes and heart tissues.a Expression of Sirt1-7 in cardiomyocytes $(n=3)$. Neonatal rat cardiomyocytes were infected with Ad-shCtrl or Ad-shPhf19 for $24 \mathrm{~h}$, and then the cells were treated with Ang II for additional $48 \mathrm{~h}$. Data are shown as mean \pm SD. $* * P<0.01$ versus Ad-shCtrl. b Phf19 overexpression repressed the expression of Sirt2 in cardiomyocytes $(n=3)$. Neonatal rat cardiomyocytes were infected with Ad-Ctrl or Ad-Phf19 for $24 \mathrm{~h}$, and then the cells were treated with Ang II for additional $48 \mathrm{~h}$. Data are shown as mean \pm SD. $* * P<0.01$ versus Ad-Ctrl. c Phf19 knockdown repressed Ang IIinduced downregulation of Sirt2 $(n=3)$. Mice with/without Phf19

\section{PHF19 Represses SIRT2 Expression Epigenetically}

Then we investigated whether PHF19 regulated SIRT2 expression epigenetically. We performed a chromatin immunoprecipitation (ChIP) assay. We detected the enrichment of PHF19, H3K36me3, and H3K27me3 at the promoter of the Sirt2 gene in cardiomyocytes (Fig. 4a and b). Phf19 knockdown increased H3K36me3 while reduced H3K27me3 enrichment at Sirt2 promoter (Fig. 4c). By contrast, $P h f 19$ overexpression significantly reduced H3K36me3 but increased H3K27me3 enrichment at the Sirt2 promoter (Fig. 4d). During cardiac hypertrophy, Ang II treatment reduced the enrichment of H3K36me3 at Sirt2 promoter in heart tissues, which was blocked by Phf19 knockdown (Fig. 4e). The effects of Ang II on H3K27me3 enrichment at the Sirt2 promoter were also blocked by the Phf19 knockdown in cardiac tissues (Fig. 4f). In addition, the enrichment of H3K36me3 and H3K27me3 at Sirt2 promoter was accomplished with PHF19 enrichment at Sirt2 promoter (Fig. 4g). Furthermore, we tested whether PHF19 regulates histone modification via H3K36me3 demethylase NO66 [13]. knockdown were subjected to Ang II treatment for four weeks. Data are shown as mean $\pm \mathrm{SD}$. ${ }^{* *} P<0.01$ versus AAV9-shCtrl, \#\#P<0.01 versus AAV9-shCtrl+Ang II. d-f SIRT2 was involved in Phf19 function in cardiomyocyte hypertrophy $(n=3)$. Neonatal rat cardiomyocytes were infected with Ad-shPhf19 or Ad-shCtrl for $24 \mathrm{~h}$, and then treated with angiotensin II (Ang II, $1 \mu \mathrm{M}$ ) for $48 \mathrm{~h}$ to induce hypertrophy in the presence/absence of SIRT2 inhibitor AGK2 $(10 \mu \mathrm{M})$ or vehicle DMSO. d Expression of Phf19 protein level. e Quantification of cardiomyocyte size. f mRNA level of Anp. $\mathbf{g}$ Protein synthesis was monitored by the incorporation of $\left[{ }^{3} \mathrm{H}\right]$-leucine. Data are shown as mean \pm SD. $* * P<0.01$ versus Ad-shCtrl + DMSO

However, we did not observe the effects of PHF19 on NO66 expression in cardiomyocytes (Supplementary Figure 4A). We also tested the enrichment of PHF19 on the promoters of other Sirtuins. The results showed that PHF19 did not bound to the promoters of other Sirtuins (Supplementary Figure 4B). Collectively, these findings demonstrated that PHF19 epigenetically regulated the expression of Sirt2 in cardiomyocyte hypertrophy.

\section{PHF19 Expression in Human Hypertrophic Hearts}

Finally, we analyzed the expression of PHF19 and SIRT2 in human hypertrophic hearts. The expression of $A N P$ and $B N P$ was significantly upregulated in hypertrophic hearts compared with the controls (Fig. 5a and d). We also observed the upregulation of PHF19 and downregulation of SIRT2 in human hypertrophic hearts (Fig. $5 \mathrm{c}$ and d). To determine the correlation of PHF19 and other genes, Spearman correlation analysis was performed. The results showed that $P H F 19$ was positively correlated with the expression level of $A N P$ and $B N P$ (Fig. 5e and f). By contrast, PHF19 was negatively 
A

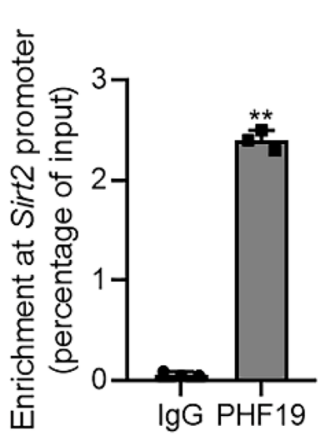

E

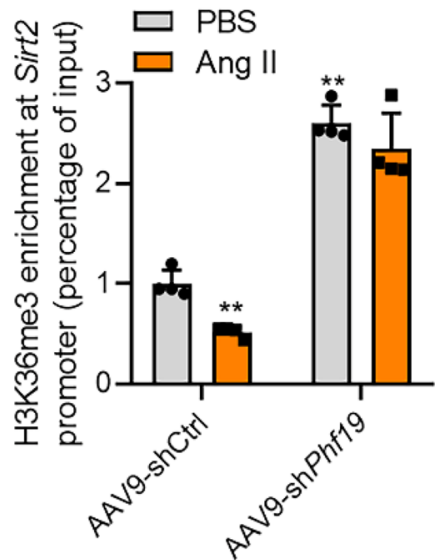

B

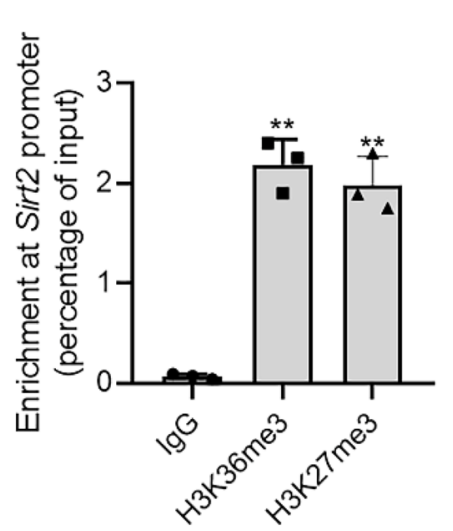

$\mathbf{F}$

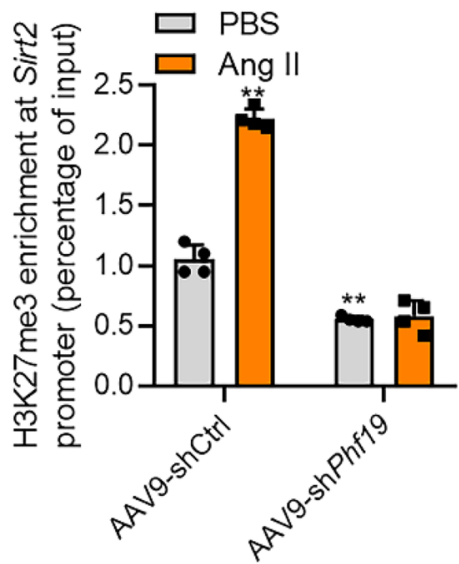

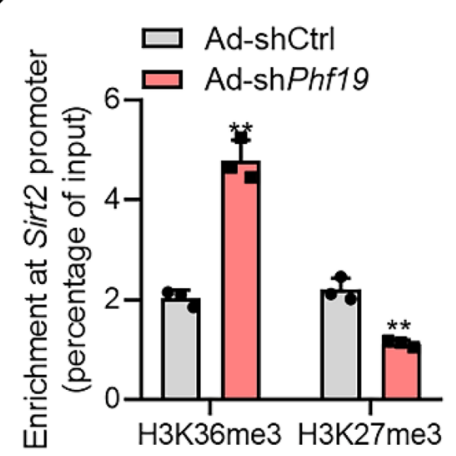

D

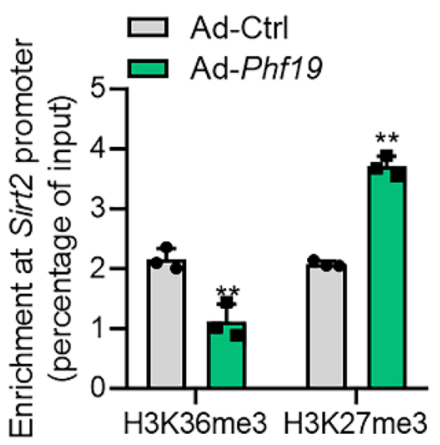

G

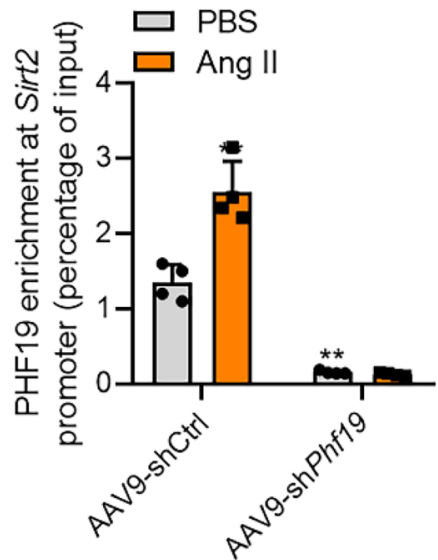

Fig. 4 Phf19 epigenetically regulates SIRT2 expression in cardiomyocytes. a PHF19 enrichment at Sirt2 promoter in rat cardiomyocytes $(n=3)$. Chromatin immunoprecipitation (ChIP) was performed with anti-IgG or anti-PHF19 antibodies with lysis from rat cardiomyocytes, followed by qRT-PCR analysis. Data are shown as mean \pm SD. $* * P<0.01$ versus $\mathrm{IgG}$. b $\mathrm{H} 3 \mathrm{~K} 27 \mathrm{me} 3$ and H3K36me3 enrichment at Sirt2 promoter $(n=3)$. ChIP was performed with anti-IgG, anti-H3K27me3, or anti-H3K36me3 antibodies with lysis from rat cardiomyocytes, followed by qRT-PCR analysis. Data are shown as mean $\pm \mathrm{SD}$. $* * P<0.01$ versus IgG. c $P h f 19$ knockdown repressed H3K27me3 enrichment and increased H3K36me3 enrichment at Sirt 2 promoter in rat cardiomyocytes $(n=3)$. Data are shown

correlated with the expression of SIRT2 in human hypertrophic hearts (Fig. 5g). Collectively, these results revealed the upregulation of PHF19 in hypertrophic hearts and the correlation with hypertrophic fetal genes and SIRT2.

\section{Discussion}

In the present study, we provided evidence that PHF19 promotes cardiac hypertrophy via epigenetically repression SIRT2 expression. With loss-of-function and gain-offunction strategies, we demonstrated that PHF19 promoted as mean $\pm \mathrm{SD}$. $* * P<0.01$ versus Ad-shCtrl. d PHF19 overexpression increased H3K27me3 enrichment and inhibited H3K36me3 enrichment at Sirt2 promoter in rat cardiomyocytes $(n=3)$. Data are shown as mean $\pm \mathrm{SD}$. $* * P<0.01$ versus Ad-Ctrl. e Phf19 knockdown repressed Ang II-induced decline in H3K36me3 enrichment at Sirt2 promoter in mouse hearts $(n=4)$. $* * P<0.01$ versus AAV9shCtrl+PBS. f Phf19 knockdown repressed Ang II-induced increase in H3K27me3 enrichment at Sirt2 promoter in mouse hearts $(n=4)$. Data are shown as mean \pm SD. $* * P<0.01$ versus AAV9-shCtrl+ PBS. g Phf19 knockdown repressed Ang II-induced increase in PHF19 enrichment at Sirt2 promoter in mouse hearts $(n=4)$. Data are shown as mean \pm SD. $* * P<0.01$ versus AAV9-shCtrl + PBS

cardiomyocyte hypertrophy and protein synthesis in vitro. We also observed that PHF19 knockdown with AAV9mediated shRNA repressed pathological cardiac hypertrophy in vivo. Mechanism study identified SIRT2 as a downstream target for PHF19, and SIRT2 downregulation was critically essential for the pro-hypertrophic roles of PHF19. PHF19 regulated the switch of the enrichment of H3K36me3 and H3K27me3 at the promoter of SIRT2 to repress the expression of SIRT2 (Fig. 6). Finally, we observed the overexpression of PHF19 and downregulation of SIRT2 in human hypertrophic hearts. 
A

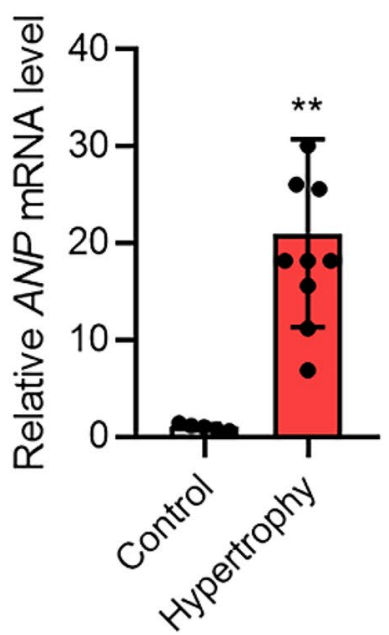

E

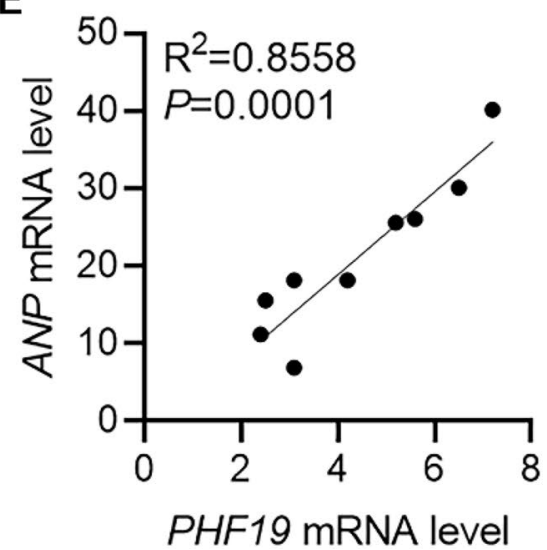

B

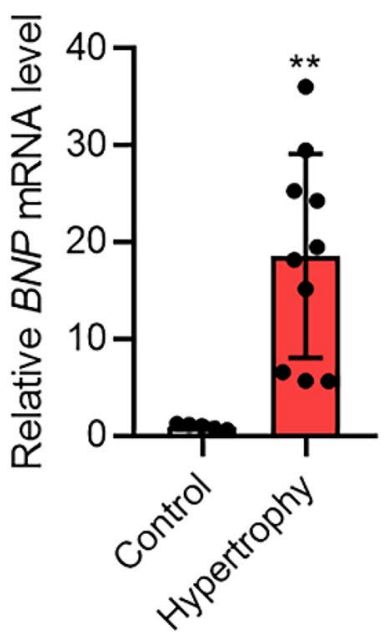

$\mathbf{F}$

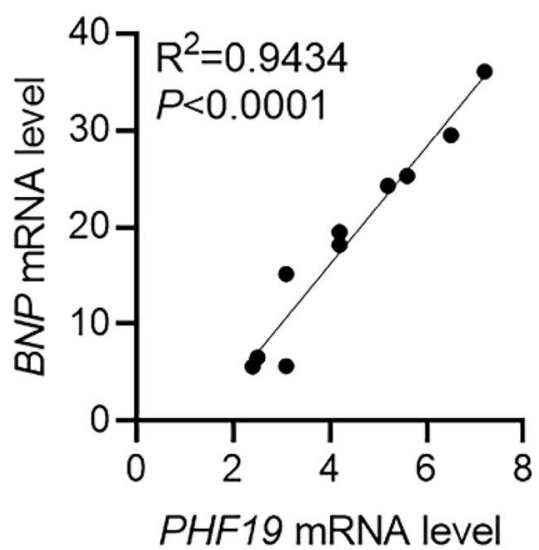

C

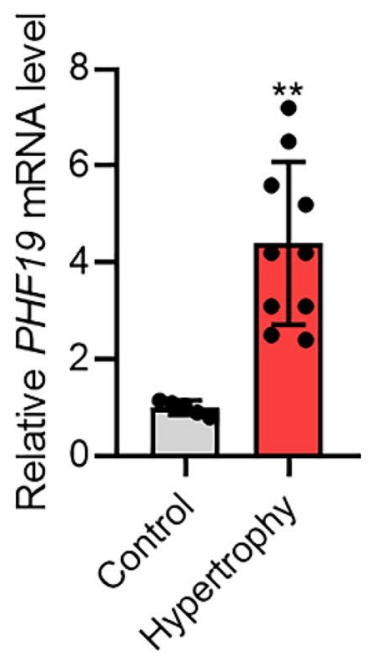

Fig. 5 PHF19 and SIRT2 expression during human and mouse cardiac hypertrophy. a-d qRT-PCR showing the expression of PHF19 (a), ANP (b), BNP (c), and SIRT2 (d) in control $(n=5)$ and hypertrophic $(n=10)$ human hearts. Data are shown as mean \pm SD. $* * P<0.01$ versus control. e Spearman correlation analysis showing PHF19 expression was positively correlated with ANP expression in human hypertrophic hearts. f Spearman correlation analysis showing PHF19 expression was positively correlated with $B N P$ expression in human hypertrophic hearts. g Spearman correlation analysis showing PHF19 expression was negatively correlated with SIRT2 expression in human hypertrophic hearts
Fig. 6 Graph showing mechanism of PHF19-SIRT2 axis in cardiac hypertrophy. Hypertrophic stress induces overexpression of PHF19, which binds SIRT2 promoter and leads to increased H3K36me3 enrichment and decreased H3K27me3 enrichment on SIRT2 promoter to repress the expression of the anti-hypertrophic factor SIRT2, and subsequently results in cardiac hypertrophy

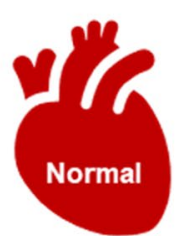

H3K36me3 H3K27me3

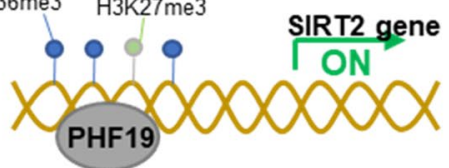

G

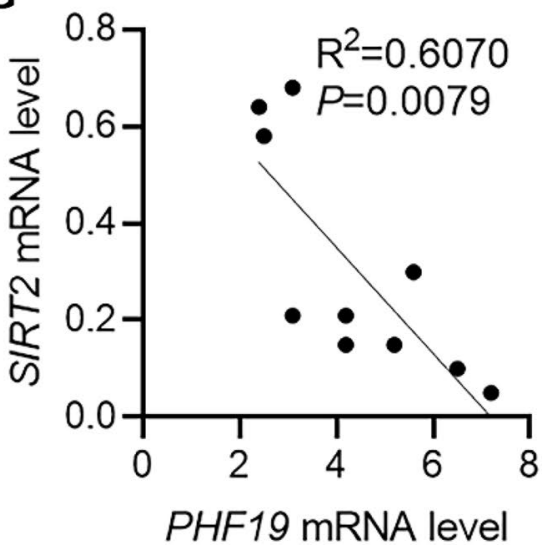


Accumulating studies have supported the notion that epigenetic regulators critically participate in the development of cardiac hypertrophy and heart failure. JMJD2A was overexpressed in hypertrophic heart tissues and promoted the development of cardiac hypertrophy via regulating H3K9me3 [9]. The histone demethylase JMJD1C regulated $\mathrm{H} 3 \mathrm{~K} 27 \mathrm{me}$ to repress CAMKK2-AMPK signaling and promote cardiac hypertrophy [10]. Besides, NSD2 promoted ventricular remodeling mediated by the regulation of H3K36me 2 [27]. However, how these histone methylations cooperate to regulate cardiac hypertrophy remains unknown. PHF19 is a subunit of the polycomb repressive complex 2 (PRC2). The interaction of H3K36me2 and H3K36me3 with PHF19 is essential for PRC2 complex activity and proper gene repression [28]. PHF19 is associated with the H3K36me3 demethylase NO66, leading to PRC2-mediated H3K27me3, loss of H3K36me3, and transcriptional silencing [13]. The critical roles of PHF19 were observed in stem cell maintenance and differentiation $[13,14,17,28]$, cancer biology $[15,16]$, reprogramming of T cells [29], and rheumatoid arthritis [30]. But the potential roles of PHF19 in the cardiovascular system remain unknown.

In this study, we observed the overexpression of PHF19 in human hypertrophic hearts, which was correlated with the overexpression of hypertrophy-related fetal genes ANP and BNP. Our loss-of-function and gain-of-function experiments demonstrated the critical pro-hypertrophic roles of PHF19 in cardiomyocytes. Of importance, we demonstrated that PHF19 promoted stress-induced cardiac hypertrophy and facilitated the decline in fraction shortening and ejection fraction. Besides, we observed that PHF19 promoted protein synthesis, a core mechanism underlying the hypertrophic growth of cardiomyocytes [7]. Overall, PHF19 acts as a pro-hypertrophic factor and may serve as a target for the treatment of cardiac hypertrophy.

Histone acetylation also participates in cardiac hypertrophy, and inhibition of HDACs was considered as a promising strategy for the treatment of cardiac hypertrophy [5]. The Sirtuin family is a core regulator for cardiac homeostasis. SIRT1 was critical for cardiac development via regulating core cardiac transcription factors, and mutations in SIRT1 lead to development defects [31, 32]. SIRT2 was reported to repress cardiac hypertrophy via diverse mechanisms, including activation of AMPK and repression of GSK3b and NFATc2 [8, 20, 21]. The mitochondrial SIRT3 and SIRT5 were cardiac protective factors that repress aging and pathological cardiac hypertrophy, whereas SIRT4 was reported as the only member as a pro-hypertrophic member [26, 33-35]. SIRT6 and SIRT7 were critical for heart development and repress cardiac hypertrophy via modulating histone acetylation and transcription factors [36]. Although the roles of Sirtuins have been identified in cardiac hypertrophy, the mechanisms underlying the regulation of Sirtuin expression during cardiac hypertrophy were not fully understood.

Here in this study, we observed that PHF19 repressed the expression of SIRT2 but not other members of Sirtuins. PHF19 bound the promoter of SIRT2 to modulate the enrichment of H3K36me 3 and H3K27me 3 at the SIRT2 promoter, subsequently leading to the suppression of SIRT2 expression. Based on previous literature $[14,17,28]$, one of the mechanisms underlying PHF19mediated SIRT2 repression is the switch of H3K36me3 and H3K27me3 at the SIRT2 promoter. PHF19 cooperated with PRC2, KDM2b/NO66 complex. PHF19 can bound H3K36me 3 directly, which leads to a reduction of H3K36me 3 by KDM2b/NO66. PHF19 also recruited $\mathrm{PRC} 2$, which increased the level of $\mathrm{H} 3 \mathrm{~K} 27 \mathrm{me} 3$, the repressor of transcription. The increased $\mathrm{H} 3 \mathrm{~K} 36 \mathrm{me} 3$ and increased H3K27me3 at the SIRT2 promoter tied the chromatin to reduce SIRT2 transcription. Our studies showed that the regulation of PHF19 on SIRT2 did not rely on NO66 expression change. Further studies are needed to determine whether PRC2 and NO66 are critically crucial for the PHF19-mediated repression of SIRT2.

The negative correlation of PHF19 and SIRT2 expressions was also observed in human hypertrophic hearts. We observed that SIRT2 was critically important for the functions of PHF19 in regulating protein synthesis and hypertrophic growth of cardiomyocytes. SIRT2 was a core deacetylase for histones, cytoplasmic and mitochondrial proteins. Thus, we identified the communication between protein methylation and acetylation within hypertrophic hearts, the imbalance of which may be one of the core mechanisms underlying cardiac hypertrophy.

In conclusion, we identified PHF19 as a pro-hypertrophy factor in pathological cardiac hypertrophy. PHF19 overexpression in the hearts leads to the imbalance of $\mathrm{H} 3 \mathrm{~K} 36 \mathrm{me} 3$ and $\mathrm{H} 3 \mathrm{~K} 27 \mathrm{me} 3$ to repress the expression of SIRT2, which is critical for the pro-hypertrophic function of PHF19. Therefore, PHF19 may serve as a target for the treatment of cardiac hypertrophy.

Supplementary Information The online version of this article (https:// doi.org/10.1007/s12012-021-09639-0) contains supplementary material, which is available to authorized users.

Acknowledgments This study was financially supported by Beijing Natural Science Foundation (7204252) and Beijing Hospitals Authority Youth Programme (QML20200603).

\section{Compliance with Ethical Standards}

Conflict of interest The authors declare that there is no conflict of interest to report. 
Open Access This article is licensed under a Creative Commons Attribution 4.0 International License, which permits use, sharing, adaptation, distribution and reproduction in any medium or format, as long as you give appropriate credit to the original author(s) and the source, provide a link to the Creative Commons licence, and indicate if changes were made. The images or other third party material in this article are included in the article's Creative Commons licence, unless indicated otherwise in a credit line to the material. If material is not included in the article's Creative Commons licence and your intended use is not permitted by statutory regulation or exceeds the permitted use, you will need to obtain permission directly from the copyright holder. To view a copy of this licence, visit http://creativecommons.org/licenses/by/4.0/.

\section{References}

1. Ding, Y. N., Tang, X., Chen, H. Z., \& Liu, D. P. (2018). Epigenetic regulation of vascular aging and age-related vascular diseases. Advances in Experimental Medicine and Biology, 1086, 55-75.

2. Chen, X.-F., Yan, L.-J., Lecube, A., \& Tang, X. (2020). Editorial: Diabetes and obesity effects on lung function. Frontiers in Endocrinology (Lausanne), 11, 462.

3. Bravo-San Pedro, J. M., Kroemer, G., \& Galluzzi, L. (2017). Autophagy and mitophagy in cardiovascular disease. Circulation Research, 120(11), 1812-1824.

4. Rawshani, A., Rawshani, A., Franzén, S., Eliasson, B., Svensson, A.-M., Miftaraj, M., et al. (2017). Mortality and cardiovascular disease in type 1 and type 2 diabetes. New England Journal of Medicine, 376, 1407-1418.

5. Nakamura, M., \& Sadoshima, J. (2018). Mechanisms of physiological and pathological cardiac hypertrophy. Nature Reviews Cardiology, 15, 387-407.

6. Chen, X.-F., Chen, X., \& Tang, X. (2020). Short-chain fatty acid, acylation and cardiovascular diseases. Clinical Science, 134, 657-676.

7. Tang, X., Li, P.-H., \& Chen, H.-Z. (2020). Cardiomyocyte senescence and cellular communications within myocardial microenvironment. Frontiers in Endocrinology (Lausanne), 11, 280.

8. Shimizu, I., \& Minamino, T. (2016). Physiological and pathological cardiac hypertrophy. Journal of Molecular and Cellular Cardiology, 97, 245-262.

9. Zhang, Q.-J., Chen, H.-Z., Wang, L., Liu, D.-P., Hill, J. A., \& Liu, Z.-P. (2011). The histone trimethyllysine demethylase JMJD2A promotes cardiac hypertrophy in response to hypertrophic stimuli in mice. The Journal of Clinical Investigation, 121, 2447-2456.

10. Yu, S., Li, Y., Zhao, H., Wang, Q., \& Chen, P. (2020). The histone demethylase JMJD1C regulates CAMKK2-AMPK signaling to participate in cardiac hypertrophy. Frontiers in Physiology, 11, 539.

11. Zhang, Q.-J., Tran, T. A. T., Wang, M., Ranek, M. J., KokkonenSimon, K. M., Gao, J., et al. (2018). Histone lysine dimethyldemethylase KDM3A controls pathological cardiac hypertrophy and fibrosis. Nature Communications, 9, 5230.

12. Morales, C. R., Li, D. L., Pedrozo, Z., May, H. I., Jiang, N., Kyrychenko, V., et al. (2016). Inhibition of class I histone deacetylases blunts cardiac hypertrophy through TSC2-dependent mTOR repression. Science Signaling, 9, ra34-ra34.

13. Brien, G. L., Gambero, G., O'Connell, D. J., Jerman, E., Turner, S. A., Egan, C. M., et al. (2012). Polycomb PHF19 binds H3K36me3 and recruits PRC2 and demethylase NO66 to embryonic stem cell genes during differentiation. Nature Structural \& Molecular Biology, 19, 1273-1281.

14. Vizán, P., Gutiérrez, A., Espejo, I., García-Montolio, M., Lange, M., Carretero, A., et al. (2020). The Polycomb-associated factor
PHF19 controls hematopoietic stem cell state and differentiation. Science Advances, 6, eabb2745.

15. Mason, M. J., Schinke, C., Eng, C. L. P., Towfic, F., Gruber, F., Dervan, A., et al. (2020). Multiple Myeloma DREAM Challenge reveals epigenetic regulator PHF19 as marker of aggressive disease. Leukemia, 34, 1866-1874.

16. Ren, Z., Ahn, J. H., Liu, H., Tsai, Y.-H., Bhanu, N. V., Koss, B., et al. (2019). PHF19 promotes multiple myeloma tumorigenicity through PRC2 activation and broad H3K27me3 domain formation. Blood, 134, 1176-1189.

17. Jain, P., Ballare, C., Blanco, E., Vizan, P., \& Di Croce, L. (2020). PHF19 mediated regulation of proliferation and invasiveness in prostate cancer cells. Elife, 9, e51373.

18. Zhou, S., Tang, X., \& Chen, H. Z. (2018). Sirtuins and insulin resistance. Frontiers in Endocrinology. (Lausanne), 9, 748.

19. Tang, X., Chen, X.-F., Wang, N.-Y., Wang, X.-M., Liang, S.-T., Zheng, W., et al. (2017). SIRT2 acts as a cardioprotective deacetylase in pathological cardiac hypertrophy. Circulation, 136, 2051-2067.

20. Sarikhani, M., Mishra, S., Maity, S., Kotyada, C., Wolfgeher, D., Gupta, M. P., et al. (2018). SIRT2 deacetylase regulates the activity of GSK3 isoforms independent of inhibitory phosphorylation. ELife, 7, e32952.

21. Sarikhani, M., Maity, S., Mishra, S., Jain, A., Tamta, A. K., Ravi, V., et al. (2018). SIRT2 deacetylase represses NFAT transcription factor to maintain cardiac homeostasis. Journal of Biological Chemistry, 293, 5281-5294.

22. Zhang, X., Lei, F., Wang, X. M., Deng, K. Q., Ji, Y. X., Zhang, Y., et al. (2020). NULP1 alleviates cardiac hypertrophy by suppressing NFAT3 transcriptional activity. Journal of the American Heart Association, 9, e016419.

23. Jia, Y.-Y., Lu, J., Huang, Y., Liu, G., Gao, P., Wan, Y.-Z., et al. (2014). The involvement of NFAT transcriptional activity suppression in SIRT1-mediated inhibition of COX-2 expression induced by PMA/Ionomycin. PLoS ONE, 9, e97999.

24. Mearini, G., Stimpel, D., Geertz, B., Weinberger, F., Krämer, E., Schlossarek, S., et al. (2014). Mybpc3 gene therapy for neonatal cardiomyopathy enables long-term disease prevention in mice. Nature Communications, 5, 5515.

25. Han, X., Chen, H., Gong, H., Tang, X., Huang, N., Xu, W., et al. (2020). Autolysosomal degradation of cytosolic chromatin fragments antagonizes oxidative stress-induced senescence. Journal of Biological Chemistry., 295(14), 4451-4463.

26. Tang, X., Chen, X.-F., Chen, H.-Z., \& Liu, D.-P. (2017). Mitochondrial sirtuins in cardiometabolic diseases. Clinical Science, 131, 2063-2078.

27. Zhou, X.-L., Zhu, R.-R., Wu, X., Xu, H., Li, Y.-Y., Xu, Q.-R., et al. (2019). NSD2 promotes ventricular remodelling mediated by the regulation of H3K36me2. Journal of Cellular and Molecular Medicine, 23, 568-575.

28. Ballaré, C., Lange, M., Lapinaite, A., Martin, G. M., Morey, L., Pascual, G., et al. (2012). Phf19 links methylated Lys36 of histone $\mathrm{H} 3$ to regulation of polycomb activity. Nature Structural \& Molecular Biology, 19, 1257-1265.

29. Ji, Y., Fioravanti, J., Zhu, W., Wang, H., Wu, T., Hu, J., et al. (2019). miR-155 harnesses Phf19 to potentiate cancer immunotherapy through epigenetic reprogramming of $\mathrm{CD} 8+\mathrm{T}$ cell fate. Nature Communications, 10, 2157.

30. Ning, F., Wang, C., Niu, S., Xu, H., Xia, K., \& Wang, N. (2018). Transcription factor Phf19 positively regulates germinal center reactions that underlies its role in rheumatoid arthritis. American Journal of Translational Research, 10, 200-211.

31. Mu, W., Zhang, Q., Tang, X., Fu, W., Zheng, W., Lu, Y., et al. (2014). Overexpression of a dominant-negative mutant of SIRT1 in mouse heart causes cardiomyocyte apoptosis and early-onset heart failure. Science China Life Sciences, 57, 915-924. 
32. Tang, X., Ma, H., Han, L., Zheng, W., Lu, Y. B., Chen, X. F., et al. (2016). SIRT1 deacetylates the cardiac transcription factor Nkx2.5 and inhibits its transcriptional activity. Scientific Reports, 6, 36576

33. Luo, Y.-X., Tang, X., An, X.-Z., Xie, X.-M., Chen, X.-F., Zhao, X., et al. (2017). Sirt4 accelerates Ang II-induced pathological cardiac hypertrophy by inhibiting manganese superoxide dismutase activity. European Heart Journal, 38, 1389-1398.

34. Sadhukhan, S., Liu, X., Ryu, D., Nelson, O. D., Stupinski, J. A., $\mathrm{Li}, \mathrm{Z}$., et al. (2016). Metabolomics-assisted proteomics identifies succinylation and SIRT5 as important regulators of cardiac function. Proceedings of the National Academy of Sciences, 113, 4320-4325.

35. Pillai, V. B., Samant, S., Sundaresan, N. R., Raghuraman, H., Kim, G., Bonner, M. Y., et al. (2015). Honokiol blocks and reverses cardiac hypertrophy in mice by activating mitochondrial Sirt3. Nature Communications, 6, 6656.

36. Sundaresan, N. R., Vasudevan, P., Zhong, L., Kim, G., Samant, S., Parekh, V., et al. (2012). The sirtuin SIRT6 blocks IGF-Akt signaling and development of cardiac hypertrophy by targeting c-Jun. Nature Medicine, 18, 1643-1650.

Publisher's Note Springer Nature remains neutral with regard to jurisdictional claims in published maps and institutional affiliations. 